\title{
Modeling of PWM Inverter-Supplied AC Drives at Low Switching Frequencies
}

\author{
T. A. Sakharuk, A. M. Stanković, G. Tadmor, Senior Member, IEEE, and G. Eirea
}

\begin{abstract}
This paper presents analytical results, numerical simulations, and experiments that quantify effects of low-switching frequency in ac drives supplied by pulsewidth modulated (PWM) power electronic inverters. Specifically, the paper re-examines models of PWM inverters in cases when the switching frequency is only an order of magnitude higher than the fundamental of the ac waveform being synthesized. While the case of permanent magnet synchronous motor is studied in detail, the presented modeling procedure is applicable to other ac drives. Analytical results derived in the paper build on related results for dc converters, and address the case of the PWM switching policy denoted as space vector modulation in industrial practice. Conventional modeling procedures assume that the PWM switching frequency is high enough so that the resulting waveform can be replaced by its first harmonic in control-oriented models. This paper provides analytical formulas in terms of the system parameters that quantify the deviations introduced by a slow PWM switching frequency.
\end{abstract}

Index Terms-AC drives, inverters, low switching frequency, pulse width modulated (PWM) systems, space vector modulation.

\section{NOMENCLATURE}

$J$

$B$

$T_{\text {load }}$

$\Phi$

$R_{d}=R_{q}=R_{a}$

$L_{d}=L_{q}=L$

$i_{d}, i_{q}$

$V_{d}, V_{q}$

$V_{d}^{*}, V_{q}^{*}$

$V_{\alpha}, V_{\beta}$

$V_{\alpha}^{*}, V_{\beta}^{*}$

$V_{1}, V_{2}, V_{3}$

$\theta$

$\omega$

$T$ $\omega_{0}$

$T_{0}$

$N$
Frequency of the basic switching PWM cycles

Period of the basic switching PWM cycles $\left(T_{0}=2 \pi / \omega_{0}\right)$.

Ratio between the (electrical) revolution period and the PWM cycle period, $N=$ $\omega_{0} / \omega=T / T_{0}$.

\section{INTRODUCTION}

V ARIABLE frequency (brushless) ac drives are increasingly replacing dc drives in a number of industrial applications due to advantages in size, reliability and efficiency. A key component of an ac drive is a power electronic converter (inverter) that takes dc input (from a rectifier or a battery), and produces a sinusoidal ac waveform, which in turn is fed to the electric motor. The fundamental frequency of this waveform is adjusted to produce the desired speed. In modern electric drives, the ac waveform comprises a number of switching cycles whose local properties (e.g., relative duration of the "on" part versus the "off" part) are varied to faithfully reproduce the desired wave shape. In polyphase (usually three phase) implementations, it is desirable to command switches in different phases in a coordinated fashion. A switching policy known as space vector modulation is particularly effective in this regard. Resulting supply waveforms have significant spectral components only at the fundamental and at integer multiples of the switching frequency. In standard installations, the separation between the fundamental and pulsewidth modulated (PWM) switching frequency is often large enough, so that in control-oriented models, it is necessary to retain only the fundamental. There are, however, practically important cases when this frequency separation does not hold. One such case is when physical characteristics of the switches do not allow for faster PWM frequencies. Another example arises in applications such as active filters where the controller aims to maximize the frequency band over which spectral content is controlled.

High switching frequency may actually cause a reduction of efficiency, e.g., in high-power industrial ac drives and railway traction applications, as switching losses in such systems account for a considerable amount of the total losses [1]. Hence, the switching frequency is often kept at low values of only few hundred hertz [2]. Reduction of switching frequency generally results in increasing current distortion, and, consequently, machine torque harmonics and higher machine losses. In active filter applications, the control goal is to cancel as many harmonics of the fundamental of a nonlinear load as possible. This often results in operation in which the ac waveform with the
Manuscript received January 25, 1999; revised March 4, 2001 and July 25, 2001. This work was supported in part by the Office of Naval Research under Grant N14-95-1-0723 and Grant N14-97-1-0704, and in part by the National Science Foundation under Grant ECS-9 410354 and Grant ECS-9 502 636. This paper was recommended by Associate Editor A. Ioinovici.

The authors are with the Department of Electrical and Computer Engineering, Northeastern University, Boston MA02115 USA.

Publisher Item Identifier S 1057-7122(02)04728-1. 


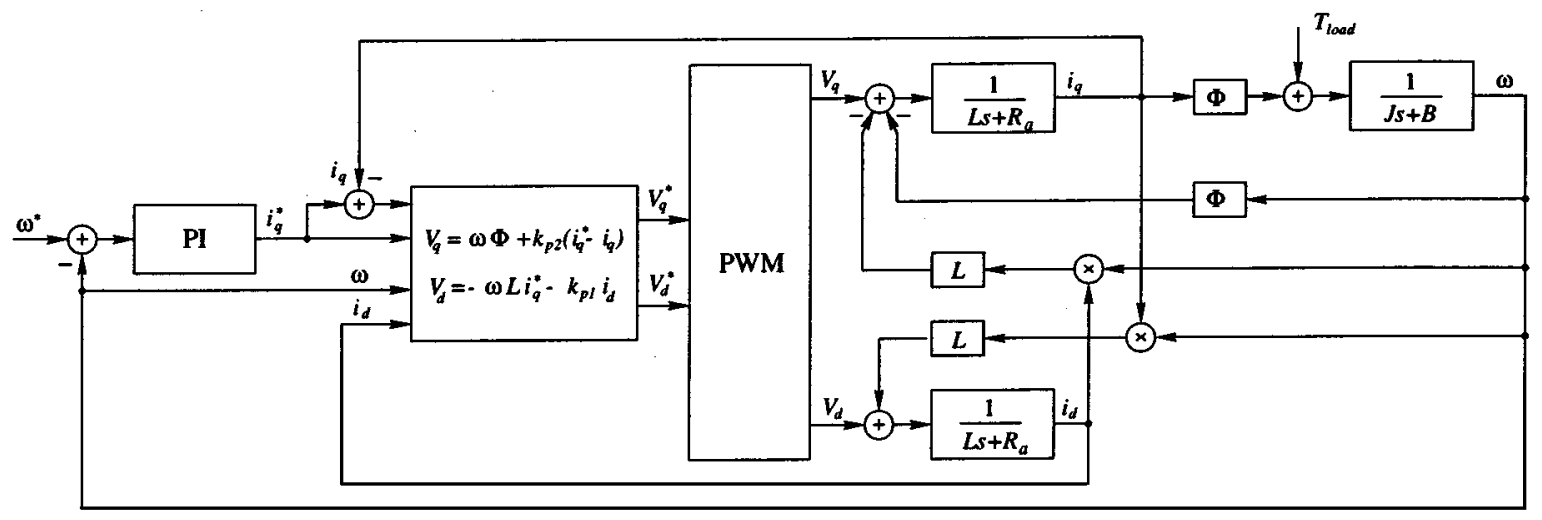

Fig. 1. AC servo motor block diagram.

highest frequency is assembled from a fairly small number of basic switching cycles.

Both classes of problems mandate a re-examination of standard control-oriented models. The common modeling approach to systems with switched power supplies [3] is to replace the pulse-width modulated power supply block with a fixed gain. It will be shown later that this approach does not provide adequate representation at low switching frequencies, as switching ripple becomes considerable. Harmonic distortion of current waveforms and different optimal modulation strategies in ac drive applications have been studied in detail during the past decade, of which [4]-[6] are few examples. However, the influence of the slow switching frequency and the current ripple on PWM gain in the closed-loop system has not been addressed. In this paper analytical formulas which quantify the effects of low switching frequency in PWM-supplied ac drives are derived. A permanent magnet (PM) synchronous motor drive will be used as a representative example of such systems; the main findings, however, are equally applicable to other ac machines, active filters and related equipment.

The rest of the paper is organized as follows. Section II introduces the model of a PM synchronous machine (PMSM), while Section III provides details about the space vector PWM that are needed in the analysis that follows. The main new results are presented in the next three sections: an expression for the open loop PWM gain in Section IV, formulas for the current ripple in Section V, and the closed loop PWM gain in Section VI. The example illustrating these results is described in Section VII, followed by a brief summary in Section VIII.

\section{MODEL OF THE PMSM}

A synchronous PM motor (with a nonsalient rotor) is described in the rotor reference frame (" $d-q$ coordinates") by the standard third-order $d-q$ model [7], depicted in Fig. 1.

The $d-q$ reference frame is the natural choice for our analysis, because all periodic quantities are constant in steady state, and model is linear at constant speed. The standard controller structure for a drive involves two loops (the inner current loop, and the outer speed loop), each with a $P I$-type controller. This setup is shown in Fig. 1, together with a feed-forward term commonly used to calculate $V_{q}^{*}$.
When the switching frequency of the PWM is fairly low, a pronounced ripple may appear in the currents. The ripple is ignored when a constant gain model of PWM is used, and a more accurate model of the PWM is needed to analyze the effects of this current ripple. Since the control of the synchronous ac motor is performed in the rotor reference frame, a model of the PWM is needed in that coordinate system. The space vector PWM switching strategy is based on reduction of a balanced three-phase system to an equivalent two-phase system, and is commonly expressed in a stationary $(\alpha-\beta)$ reference frame.

The transformation between the rotor reference frame and the phase quantities (via the $\alpha-\beta$ frame), is given by

$$
\begin{aligned}
{\left[\begin{array}{l}
V_{d} \\
V_{q}
\end{array}\right] } & =\left[\begin{array}{cc}
\cos \theta & \sin \theta \\
-\sin \theta & \cos \theta
\end{array}\right]\left[\begin{array}{l}
V_{\alpha} \\
V_{\beta}
\end{array}\right] \\
& =\left[\begin{array}{cc}
\cos \theta & \sin \theta \\
-\sin \theta & \cos \theta
\end{array}\right]\left[\begin{array}{ccc}
1 & \cos \frac{2 \pi}{3} & \cos \frac{2 \pi}{3} \\
0 & -\sin \frac{2 \pi}{3} & \sin \frac{2 \pi}{3}
\end{array}\right]\left[\begin{array}{l}
V_{1} \\
V_{2} \\
V_{3}
\end{array}\right]
\end{aligned}
$$

which is invertible if we assume balanced operation, i.e., $V_{1}+$ $V_{2}+V_{3}=0$.

This leads to the following transformation between the phase quantities and the rotor reference frame, expressed in complex notation

$$
V_{d}+j V_{q}=e^{-j \theta} V_{1}+e^{-j(\theta+2 / 3 \pi)} V_{2}+e^{-j(\theta-2 / 3 \pi)} V_{3}
$$

When the switching frequency of the PWM is fairly low and the mechanical time constant of the motor is relatively large, an approximate solution for the currents $i_{d}, i_{q}$ can be found as follows: the voltage output $V_{d}$ and $V_{q}$ will be approximated by piecewise constant functions, and the currents $i_{d}$ and $i_{q}$ by piecewise linear functions. Approximations of $V_{d}^{*}$ and $V_{q}^{*}$ can then be obtained from analytical solutions for currents, using the assumption that the system is close to steady state. This approach will allow us to estimate the magnitude of the current ripple, and to quantify deviations of the actual PWM gain from the idealized one.

\section{Voltage Space Vector PWM}

There exists a number of PWM methods for variable frequency voltage-sourced inverters. Among these, PWM strategies based on the concept of voltage space vectors [9], [10] have 


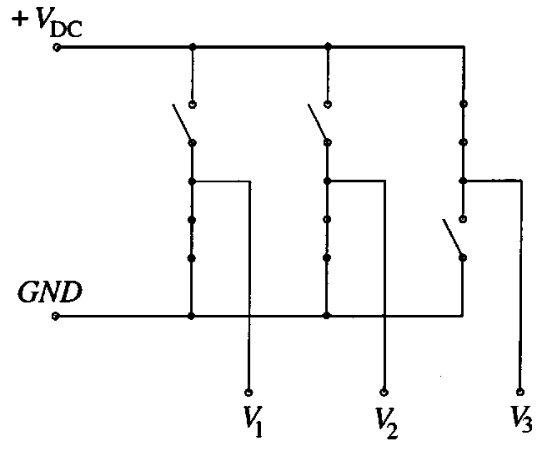

Fig. 2. Inverter for a three-phase ac motor.

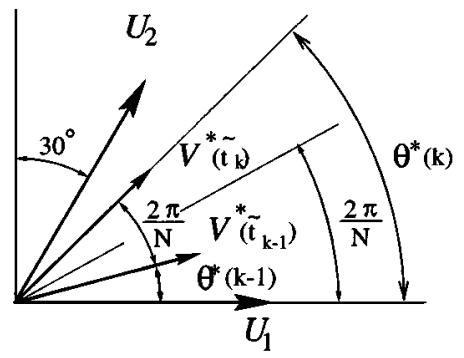

Fig. 3. Explanation of the definition of reference input voltage vectors. In this example $N=\left(T / T_{0}\right)=12$ and $t_{s}=0$.

established numerous advantages in practice. They are capable of producing the highest achievable fundamental output voltage with low harmonic distortion of the output current, and are well suited for microprocessor implementation [8].

Space vector modulation is typically described in the $\alpha-\beta$ plane [11], as the desired three-phase sinusoidal output voltages correspond to a circular path. Assume that the waveform produced by the inverter consists of basic switching cycles with frequency $\omega_{0}=2 \pi / T_{0}$, which is an integer multiple to the slower fundamental stator frequency $\omega=2 \pi / T$, where $N=\omega_{0} / \omega=$ $T / T_{0}$. Practical reasons for maintaining integer value of $N$ are outlined in [12]. The PWM inverter shown in Fig. 2 attempts to reproduce (in a given basic switching cycle $(k-(1 / 2)) T_{0}<$ $\left.t \leq(k+(1 / 2)) T_{0}\right)$ the voltage vector $V^{*}(t)=V_{\alpha}^{*}+j V_{\beta}^{*}$ demanded by the current controller. The best approximation would be achieved if the reference voltage $V^{*}(t)$ were sampled at $\tilde{t}_{k}=k T_{0}+t_{s}\left(\left|t_{s}\right| \leq T_{0} / 2\right)$ so that the sampled value $V^{*}\left(\tilde{t}_{k}\right)$ is equal to the average voltage within the present subinterval $\left[t_{k}-T_{0} / 2, t_{k}+T_{0} / 2\right]$. This is not possible, however, because the switching sequence is to be determined by the time $(k-(1 / 2)) T_{0}$. For this reason, $V^{*}\left(\tilde{t}_{k}\right)$ will be approximated by a phase-shifted quantity $V^{*}\left(\tilde{t}_{k-1}\right) e^{j 2 \pi T_{0} / T}=V_{\mathrm{dc}} R^{*}(k) e^{j \theta^{*}(k)}$ (see Fig. 3), where $V_{\mathrm{dc}}$ is the inverter dc link voltage, and $R^{*}$ and $\theta^{*}$ are the magnitude and the angle of the reference vector

$$
\begin{aligned}
R^{*}(k) & =\left(\sqrt{\left(V_{d}^{*}\left(\tilde{t}_{k-1}\right)\right)^{2}+\left(V_{q}^{*}\left(\tilde{t}_{k-1}\right)\right)^{2}}\right) / V_{\mathrm{dc}} \\
\theta^{*}(k) & =\angle V^{*}\left(\tilde{t}_{k-1}\right)+2 \pi \frac{T_{0}}{T} .
\end{aligned}
$$

There exist important practical constraints on switching patterns that are implemented by the inverter-none of the three inverter phases ("legs") is allowed to short-circuit the dc link voltage, and contours must exist so that all phase currents are continuous. The switching state of each leg is indicated by a

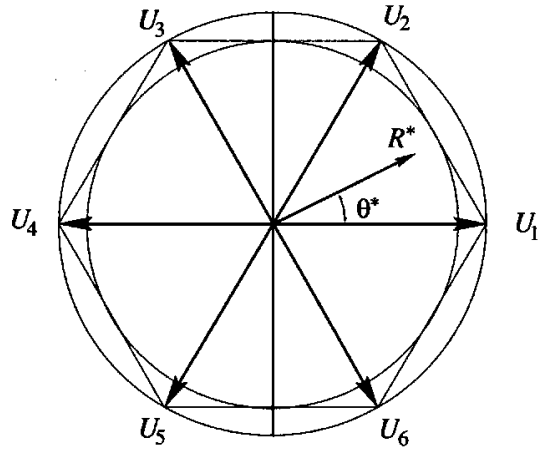

Fig. 4. Voltage vectors created by the switching states of the inverter.

binary variable 0 (top switch off) or 1 (top switch on). The switching state of the inverter may thus be described by three numbers characterizing the eight allowed states. States 000 and 111 correspond to the situation when all motor terminals are connected to the upper or lower rail of the dc link circuit, thus being shorted. These states thus correspond to six active vectors that form a hexagon shown in Fig. 4 [13] and two null (zero) vectors located at the origin. In our notation $U_{1}$ corresponds to the state $(011), U_{2}$ to $(010)$, etc.; for example, the switching state $U_{6}(001)$ is depicted in Fig. 2. The three phase voltages $V_{1}, V_{2}, V_{3}$ produced by the inverter and applied to the motor terminals approximate the desired three phase sinusoidal voltages. Since the commanded reference voltage vector $V^{*}(t)$ is typically not coinciding with one of the available 8 voltage vectors in any switching period, the required (sampled) reference voltage vector is realized by a switching sequence comprising the directly adjacent lead and lag active vectors $U_{\mathrm{ld}}$ and $U_{\mathrm{lg}}=U_{\mathrm{ld}} e^{-j \pi / 3}$. The durations $t_{\mathrm{ld}}$ and $t_{\mathrm{lg}}$ of the two adjacent vectors are determined in such a way that the actual output voltage vector, when averaged over one switching cycle, coincides with the reference vector $V^{*}(t)$. These durations are computed from the following projection equations, derived directly from Fig. 4

$$
\begin{aligned}
& \frac{t_{\mathrm{ld}}(k)}{T_{0}}=\frac{2 R^{*}(k)}{\sqrt{3}} \sin \left(\theta^{*}(k)\right) \\
& \frac{t_{\mathrm{lg}}(k)}{T_{0}}=R^{*}(k) \cos \left(\theta^{*}(k)\right)-\frac{R^{*}(k)}{\sqrt{3}} \sin \left(\theta^{*}(k)\right)
\end{aligned}
$$

where $\theta^{*}(k)$ is the angle of the reference voltage within the first sector $\theta^{*}(k) \in[0, \pi / 3]$. For angles $\theta^{*}$ in the other five sectors, the same equations can be obtained by substituting $\theta^{*} \bmod (\pi / 3)$ instead of $\theta^{*}$ into (4), (5). The sum $t_{\mathrm{ld}}+t_{\mathrm{lg}}$ is smaller than $T_{0}$ as long as the reference vector $V^{*}(t)$ lies inside the inner circle shown in Fig. 4, implying that the two active vectors occupy only part of a switching cycle; the rest is filled out by zero vectors

$$
\frac{t_{z}(k)}{T_{0}}=1-\frac{t_{\mathrm{ld}}+t_{\mathrm{lg}}}{T_{0}} .
$$

The magnitude $\left|V_{d}^{*}+j V_{q}^{*}\right|$ of the reference voltage in the range up to $(\sqrt{3} / 2) V_{\mathrm{dc}}$ can be reproduced by this method. Further increase of the reference voltage leads to saturation of the PWM [12]. The following normalized notation $\tau_{1}(k)=\left(t_{\mathrm{ld}}(k)\right) /\left(T_{0}\right), \tau_{2}(k)=\left(t_{\mathrm{lg}}(k)\right) /\left(T_{0}\right)$ and $\tau_{z}=\left(t_{z}(k)\right) /\left(T_{0}\right)=1-\tau_{1}(k)-\tau_{2}(k)$ will be used, where 
$\tau_{1}, \tau_{2}$ and $\tau_{z}$ are duty ratios of leading $U_{\mathrm{ld}}$, lagging $U_{\mathrm{lg}}$ and null vectors. The ability to apportion the zero-vector time around two active vectors represents a degree of freedom that is used to improve the performance of space vector modulation with respect to auxiliary issues like common-mode currents.

\section{A. Switching Pattern}

The choice of either one of the two zero vectors per switching cycle allows various combinations of the sequence of states. In the most commonly employed regular sampling mode, each (electrical) period of rotation $T$ is divided into six sectors and each sector into $N / 6$ segments (PWM cycles) representing individual switching intervals.

Several ways are used, in practice, to order the active and zero vectors within a basic switching cycle. We concentrate on one of the frequently used patterns that is denoted as symmetric or centered PWM switching sequence [14]. In this vector PWM strategy, the inverter state is changed four times within each segment. The state sequence within a given sector is as shown in (7) at the bottom of the page where $U_{\mathrm{ld}}$ and $U_{\mathrm{lg}}$ are the leading and lagging adjacent to the reference $V^{*}$ vectors. For example, if $V^{*}$ is in the first sector i.e., $0<\theta^{*}<\pi / 3$, then $U_{\mathrm{ld}}=U_{2}$ and $U_{\mathrm{lg}}=U_{1}$. For this switching pattern there are four switching instances

$$
\begin{array}{lll}
t_{k}-d_{1}(k) T_{0} & t_{k}-d_{2}(k) T_{0}, & t_{k}+d_{2}(k) T_{0} \\
t_{k}+d_{1}(k) T_{0} &
\end{array}
$$

within every time interval $\left[t_{k}-T_{0} / 2, t_{k}+T_{0} / 2\right]$, where $t_{k}$ is the midpoint of the time interval, and $d_{1}(k)=\left(\tau_{1}+\tau_{2}\right) / 2, d_{2}(k)=$ $\tau_{1} / 2$ can be computed from (4), (5) as

$$
\begin{aligned}
& d_{1}(k)=\frac{R^{*}(k)}{\sqrt{3}} \cos \left(\theta^{*}(k)-\frac{\pi}{6}\right) \\
& d_{2}(k)=\frac{R^{*}(k)}{\sqrt{3}} \sin \left(\theta^{*}(k)\right) .
\end{aligned}
$$

where $\theta^{*} \in[0, \pi / 3]$. For angles $\theta^{*}$ within other five sectors the same equations can be obtained by substitution $\theta^{*} \bmod (\pi / 3)$ into (4), (5) instead of $\theta^{*}$, so that $d_{1}, d_{2}$ are $(\pi / 3)$-periodic as functions of $\theta^{*}$.

\section{B. Analytical Expression for $d-q$ Voltages}

Assume for now that the system is in a steady state, so that reference voltages in $d-q$ coordinates $V_{d}^{*}$ and $V_{q}^{*}$ are constant. Then the actual 3-phase PWM output line voltages $V_{1}(t), V_{2}(t)$ and $V_{3}(t)$ are $T$-periodic functions; moreover there is a constant phase delay among these waveforms. It is then straightforward to show that $V_{d}(t)+j V_{q}(t)$ is a $T / 6$ periodic function. Without loss of generality, suppose that the interval of interest corresponds to $[0, \pi / 3]$ in angular coordinates, so that vectors $U_{1}=U_{\mathrm{lg}}=\left[\begin{array}{lll}0 & 1 & 1\end{array}\right]^{\prime}$ and $U_{2}=U_{\mathrm{ld}}=\left[\begin{array}{lll}0 & 1 & 0\end{array}\right]^{\prime}$ are used to produce the switching sequence.

The interval $[0, T / 6]$ consists of $N / 6$ subintervals, each of length $T_{0}$, where $T_{0}$ is the inverter basic switching cycle. These subintervals have similar structure, as the same vectors $U_{\mathrm{ld}}, U_{\mathrm{lg}}$ are used, but durations $t_{\mathrm{ld}}$ and $t_{\mathrm{lg}}$ given by (4) and (5) are different. Each subinterval can be divided into five smaller intervals within which the line voltages are constant (zero, lag, lead, lag, zero). From this observation, and using (2), it can be concluded that $\left(V_{d}(t)+j V_{q}(t)\right) / V_{\mathrm{dc}}$ equals 0 during zero intervals, $e^{-j(2 t / T+2 / 3) \pi}$ during the lead interval, and $e^{-j(2 t / T+2 / 3) \pi}+e^{-j(2 t / T-2 / 3) \pi}$ during the lag intervals. Recall that $e^{-j(2 t / T+2 / 3) \pi}+e^{-j(2 t / T-2 / 3) \pi}=-e^{-j(2 t / T) \pi}$. Finally, $V_{d}$ and $V_{q}$ are piecewise sinusoidal functions as shown in the equation at the bottom of the pagewhere $n=k \bmod (N / 6)$, and $\theta_{0}$ is an angle between reference voltage in $d-q$ coordinate and real axis, which can be determine as a difference between rotor angle $\theta$ and reference voltage angle $V_{\alpha}^{*}+j V_{\beta}^{*}$ (in the $\alpha-\beta$ coordinate system) $\theta_{0}=\angle\left(V_{d}^{*}+j V_{q}^{*}\right)=\theta^{*}-\theta$ (see (1)). In steady state both $\theta^{*}$ and $\theta$ are changing with constant speed $\omega$, so $\theta_{0}$ is constant.

\section{OPEN-LOOP PWM GAIN}

The averaged open-loop PWM gain is defined as a ratio of $T$-averaged output PWM voltage $\bar{V}_{d}+j \bar{V}_{q}$ to the constant input

$$
K_{\mathrm{PWM}}^{\mathrm{oll}}=\frac{\bar{V}_{d}+j \bar{V}_{q}}{V_{d}^{*}+j V_{q}^{*}}
$$

where $V_{d}^{*}+j V_{q}^{*}$ is the constant reference. Ideally, the open-loop gain is equal to 1 , but this may not be the case due to the vector space PWM implementation strategy.

\begin{tabular}{|c||c|c|c|c|c|}
\hline Voltage vector & Zero & $U_{\mathrm{lg}}$ & $U_{\mathrm{ld}}$ & $U_{\mathrm{lg}}$ & Zero \\
\hline Duration & $t_{z} / 2$ & $t_{\mathrm{lg}} / 2$ & $t_{\mathrm{ld}}$ & $t_{\mathrm{lg}} / 2$ & $t_{z} / 2$ \\
\hline
\end{tabular}

$$
\frac{V_{d}\left(t_{k}+\gamma T_{0}\right)+j V_{q}\left(t_{k}+\gamma T_{0}\right)}{V_{\mathrm{dc}}}=\left\{\begin{array}{l}
0, \\
-e^{-j\left(\frac{2 \pi}{N}\left(\frac{2 n+1}{2}+\gamma\right)-\theta_{0}\right)} \\
e^{-j\left(\frac{2 \pi}{N}\left(\frac{2 n+1}{2}+\gamma\right)+\frac{2}{3} \pi-\theta_{0}\right)} \\
-e^{-j\left(\frac{2 \pi}{N}\left(\frac{2 n+1}{2}+\gamma\right)-\theta_{0}\right)} \\
0
\end{array}\right.
$$

$$
\begin{array}{ll}
\text { if } \gamma \in\left[-\frac{1}{2},-d_{1}(n)\right) & \text { Zero Vector } \\
\text { if } \gamma \in\left[-d_{1}(n),-d_{2}(n)\right) & \text { Lag Vector } \\
\text { if } \gamma \in\left[-d_{2}(n), d_{2}(n)\right) & \text { Lead Vector } \\
\text { if } \gamma \in\left[d_{2}(n), d_{1}(n)\right) & \text { Lag Vector } \\
\text { if } \gamma \in\left[d_{1}(n), \frac{1}{2}\right) & \text { Zero Vector }
\end{array}
$$


Since the output voltage of the PWM periodic with period $T / 6$, it is suffices to consider one such period. Then the averaged PWM output voltage can be obtained as

$$
\bar{V}_{d}+j \bar{V}_{q}=\frac{6}{N} \sum_{n=0}^{N / 6-1}\left(S_{d}(n)+j S_{q}(n)\right)
$$

where

$$
S_{d}(n)+j S_{q}(n)=\frac{1}{T_{0}} \int_{-T_{0} / 2+t_{k}}^{T_{0} / 2+t_{k}}\left(V_{d}(t)+j V_{q}(t)\right) d t
$$

and $n=k \bmod (N / 6)$ is the index of current switching cycle within $\pi / 3$ interval. To evaluate the integral, the interval $\left[t_{k}-\right.$ $\left.T_{0} / 2, t_{k}+T_{0} / 2\right]$ is divided into 5 subintervals according to the switching pattern (8), where $\theta^{*}(n)=(2 n+1) / N \pi$. The integral (12) is equal to sum of five integrals $S_{d}^{\ell}(n)+j S_{q}^{\ell}(n), \ell=$ $0, \ldots, 4$, where $S^{0}=0$ and $S^{4}=0$ and

$$
\begin{aligned}
& S_{d}^{1}(n)+j S_{q}^{1}(n)=V_{\mathrm{dc}} \int_{-d_{1}(n)}^{-d_{2}(n)}-e^{-j\left(\frac{2 \pi}{N}\left(\frac{2 n+1}{2}+s\right)-\theta_{0}\right)} d s \\
& S_{d}^{2}(n)+j S_{q}^{2}(n)=V_{\mathrm{dc}} \int_{-d_{2}(n)}^{d_{2}(n)} e^{-j\left(\frac{2 \pi}{N}\left(\frac{2 n+1}{2}+s\right)+\frac{2}{3} \pi-\theta_{0}\right)} d s \\
& S_{d}^{3}(n)+j S_{q}^{3}(n)=V_{\mathrm{dc}} \int_{d_{2}(n)}^{d_{1}(n)}-e^{-j\left(\frac{2 \pi}{N}\left(\frac{2 n+1}{2}+s\right)-\theta_{0}\right)} d s .
\end{aligned}
$$

The final result is a fairly complicated algebraic expression, nonlinear in terms of $R^{*}$. The Taylor series expansion produces the following approximate solution:

$$
\begin{aligned}
\frac{\bar{V}_{d}(n)}{V_{\mathrm{dc}}}= & R^{*} \cos \left(\theta_{0}\right)-\left(R^{*} \frac{2}{\sqrt{3}}\right)^{3} \\
& \times\left(\frac{\pi}{N}\right)^{2} \frac{1}{6}\left[\cos \left(\frac{2 n+1}{N} \pi-\theta_{0}\right)\right. \\
& \times\left(-\sin \left(\frac{2 n+1}{N} \pi\right)^{3}+\sin \left(\frac{2 n+1}{N} \pi+\frac{\pi}{3}\right)^{3}\right) \\
& \left.+\sin \left(\frac{2 n+1}{N} \pi-\theta_{0}+\frac{\pi}{6}\right) \sin \left(\frac{2 n+1}{N} \pi\right)^{3}\right] \\
& +O\left(\left(R^{*}\right)^{5}\right) \\
\frac{\bar{V}_{q}(n)}{V_{\mathrm{dc}}}= & R^{*} \sin \left(\theta_{0}\right)-\left(R^{*} \frac{2}{\sqrt{3}}\right)^{3} \\
& \times\left(\frac{\pi}{N}\right)^{2} \frac{1}{6}\left[\sin \left(\frac{2 n+1}{N} \pi-\theta_{0}\right)\right. \\
& \times\left(\sin \left(\frac{2 n+1}{N} \pi\right)^{3}-\sin \left(\frac{2 n+1}{N} \pi+\frac{\pi}{3}\right)^{3}\right) \\
& \left.+\cos \left(\frac{2 n+1}{N} \pi-\theta_{0}+\frac{\pi}{6}\right) \sin \left(\frac{2 n+1}{N} \pi\right)^{3}\right] \\
& +O\left(\left(R^{*}\right)^{5}\right)
\end{aligned}
$$

Here, the first term is linear on $R^{*}$ and represents ideal output of the PWM. Note that $\left(R^{*}(2 / \sqrt{3})\right)$ is always less then 1 . The $\ell$-th term is smaller than $(\pi / N)^{2 \ell} 1 /((2 \ell+1) !)$ and decreases rapidly with $\ell$.
Finally, we conclude that the open-loop gain of the PWM

$$
K_{\mathrm{PWM}}^{\mathrm{ol} . \mathrm{l}}=\frac{\bar{V}_{d}+j \bar{V}_{q}}{V_{d}^{*}+j V_{q}^{*}}=\frac{\bar{V}_{d}+j \bar{V}_{q}}{V_{\mathrm{dc}} R^{*} e^{j \theta_{0}}} \approx 1 .
$$

The error in the gain of the PWM is in order of $1 / 6(2 / \sqrt{3})^{3}(\pi / N)^{2}$, and does not depend on the absolute switching frequency $\omega_{0}=2 \pi / T_{0}$, but depends on the relative switching frequency $N$, i.e., on the number of basic switching cycles per period of the fundamental.

\section{A. Example}

Let $N=12$ and $V_{\mathrm{dc}}=1$, then within every $T / 6$ time interval there are two subintervals $n=0,1$ with

$$
\begin{aligned}
& d_{1}(0)=R^{*} \frac{\sqrt{3}+1}{2 \sqrt{6}}=0.5577 R^{*} \\
& d_{2}(0)=R^{*} \frac{\sqrt{3}-1}{2 \sqrt{6}}=0.1494 R^{*}
\end{aligned}
$$

for the first one, and

$$
\begin{aligned}
& d_{1}(1)=R^{*} \frac{\sqrt{3}+1}{2 \sqrt{6}}=0.5577 R^{*} \\
& d_{2}(1)=R^{*} \frac{1}{\sqrt{6}}=0.4082 R^{*}
\end{aligned}
$$

for the second one.

The fifth-order Taylor polynomial approximation to $\bar{V}_{d}$ and $\bar{V}_{q}$ is:

$$
\begin{aligned}
& \bar{V}_{d} \approx R^{*} \cos \left(\theta_{0}\right)-1.4730 \cdot 10^{-2} R^{* 3} \cos \left(\theta_{0}-0.3108\right) \\
& \bar{V}_{q} \approx R^{*} \sin \left(\theta_{0}\right)-1.4730 \cdot 10^{-2} R^{* 3} \sin \left(\theta_{0}-0.3108\right) .
\end{aligned}
$$

The complex gain is

$$
K_{\mathrm{PWM}}^{\mathrm{oll}} \approx 1.0-1.4730 \cdot 10^{-2} R^{* 2} e^{-j 0.3108} .
$$

For this particular example, the averaged PWM output voltage differs from the ideal by less then $1.5 \%$.

\section{Estimation of the CuRRent RipPle Magnitude}

In this section, a formula for the magnitude of the current ripple will be obtained using a piecewise linear approximation for the current. To find the currents $i_{d}$ and $i_{q}$, the voltages $V_{d}^{f}=$ $V_{d}+L i_{q} \omega$ and $V_{q}^{f}=V_{q}-\Phi \omega-L i_{d} \omega$ are applied to motor phases represented by the first-order system $1 /\left(L s+R_{a}\right)$. In evaluating $V_{d}^{f}$ and $V_{q}^{f}$ we neglect ripple in $i_{d}, i_{q}$, and $\omega$; this can be justified by low-pass filtering provided by the drive and the load, and by the relatively small magnitude of corresponding terms when compared with $V_{d}, V_{q}$ (PWM output voltage). With this assumption, the currents waveforms will depend only on $V_{d}$ and $V_{q}$; other terms in $V_{d}^{f}$ and $V_{q}^{f}$ will contribute to the constant dc value of the currents. Thus, for the purpose of ripple evaluation, voltages $V_{d}, V_{q}$ can be used instead of $V_{d}^{f}$ and $V_{q}^{f}$. Voltages $V_{d}, V_{q}$ are piecewise sinusoidal functions as shown in Section IV.B. To find simplified solutions for currents, voltages $V_{d}, V_{q}$ will be approximated by piecewise-constant functions 

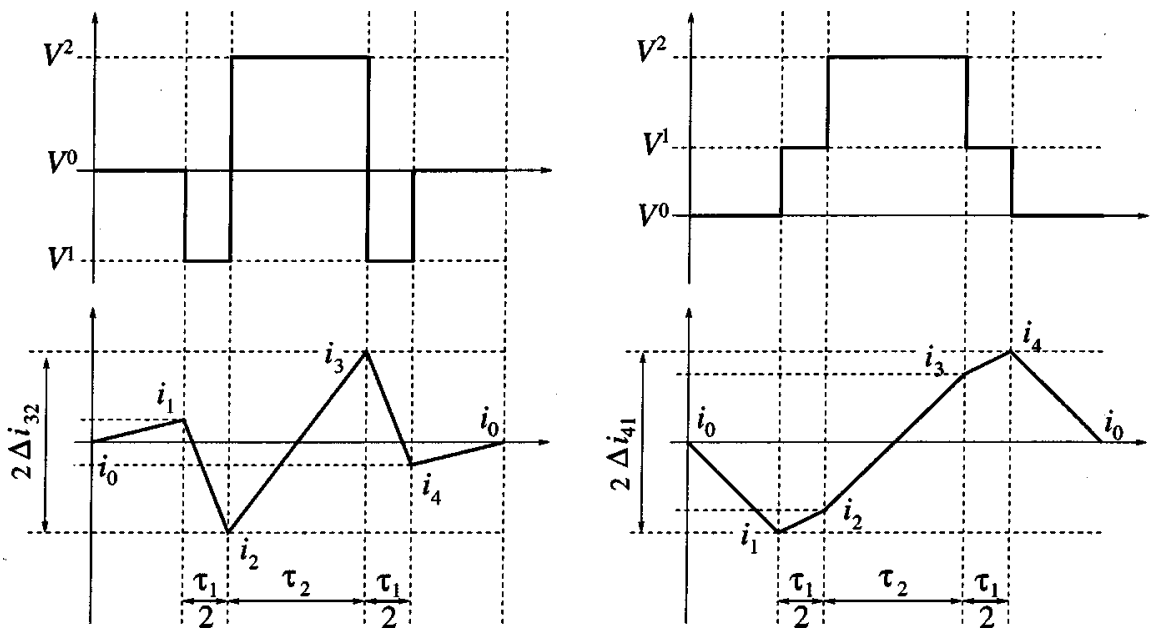

Fig. 5. Typical voltages and corresponding currents waveform patterns for one basic inverter cycle $T_{0}$. Magnitude of the current ripple is defined differently for the two patterns: $\Delta i_{3,2}=\left(i_{3}-i_{2}\right) / 2$ and $\Delta i_{4,1}=\left(i_{4}-i_{1}\right) / 2$.

such that averaged values in every time interval between switchings are preserved.

The following discussion applies equally to $d$ and $q$ axis; to simplify notations the subindices $d$ and $q$ are omitted if the same equation applies to both axes-for example, depending on the context, $V$ stands for $V_{d}$ or $V_{q}$.

Let the current at the time $t_{0}$ be $i\left(t_{0}\right)$, and let the constant voltage $V$ be applied to the motor phase represented by the first-order system $1 /\left(L s+R_{a}\right)$; then the current at $t_{0}+\Delta t$ is approximately

$$
i\left(t_{0}+\Delta t\right)=\frac{V-R_{a} i\left(t_{0}\right.}{L} \Delta t+i\left(t_{0}\right) .
$$

From $\left|R_{a}\left(i\left(t_{0}+\Delta t\right)-i\left(t_{0}\right)\right)\right| \ll|V|$, further simplification can be obtained

$$
i\left(t_{0}+\Delta t\right)=\frac{V-R_{a} \bar{i}}{L} \Delta t+i\left(t_{0}\right)
$$

where $\bar{i}$ is an averaged current. Typical voltage and corresponding current waveforms for switching pattern (7) are shown in Fig. 5.

The expressions for current ripple for the two typical current patterns are obtained from a Taylor series expansion (the details are in the Appendix A)

$$
\begin{aligned}
\Delta i_{4,1}^{d}= & M R^{*} \cos \left(\theta_{0}\right)\left(1-\frac{2}{\sqrt{3}} R^{*} \cos \left(\frac{2 n+1}{N} \pi-\frac{\pi}{6}\right)\right) \\
\Delta i_{4,1}^{q}= & M R^{*} \sin \left(\theta_{0}\right)\left(1-\frac{2}{\sqrt{3}} R^{*} \cos \left(\frac{2 n+1}{N} \pi-\frac{\pi}{6}\right)\right) \\
\Delta i_{3,2}^{d}= & M \frac{2}{\sqrt{3}} R^{*} \sin \left(\frac{2 n+1}{N} \pi\right)\left(\operatorname { s i n } \left(\frac{2 n+1}{N} \pi\right.\right. \\
& \left.\left.-\theta_{0}+\frac{\pi}{6}\right)-R^{*} \cos \left(\theta_{0}\right)\right) \\
\Delta i_{3,2}^{q}= & M \frac{2}{\sqrt{3}} R^{*} \sin \left(\frac{2 n+1}{N} \pi\right)\left(\operatorname { c o s } \left(\frac{2 n+1}{N} \pi\right.\right. \\
& \left.\left.-\theta_{0}+\frac{\pi}{6}\right)-R^{*} \sin \left(\theta_{0}\right)\right)
\end{aligned}
$$

where

$$
M=\frac{\pi}{N \omega} \frac{V_{\mathrm{dc}}}{L}=\frac{T_{0}}{2} \frac{V_{\mathrm{dc}}}{L} .
$$

Note that $\Delta i_{4,1}=M\left(V_{d}^{*}+j V_{q}^{*}\right) /\left(V_{\mathrm{dc}}\right)\left(1-\tau_{1}-\tau_{2}\right)$. The largest ripple occurs when the reference voltage vector is aligned with $d$ or $q$ axis, i.e., $\theta_{0}=(\pi / 2) \ell, \ell=0, \pm 1, \pm 2$. Let $\theta_{0}=0$ or $\theta_{0}=\pi$, then $\Delta i_{4,1}^{q}=0$ and $\Delta i_{4,1}^{d}$ is a second degree polynomial in $R^{*}$ with the maximum

$$
\Delta i_{41, \max }^{d}=\frac{M}{\frac{8}{\sqrt{3}} \cos \left(\frac{2 n+1}{N} \pi-\frac{\pi}{6}\right)} .
$$

For different subintervals, the absolute maximum $\max _{n}$ $\left[\Delta i_{41, \max }^{d}(n)\right]=M / 4$ is achieved in the first $n=0$ and the last $n=N / 6-1$ subintervals.

From (26) and (27), it follows that when $V_{d}^{*}+j V_{q}^{*}$ is aligned with either the $d$ or the $q$ axis, the ripple magnitude $\Delta i_{4,1}^{d}$ or $\Delta i_{4,1}^{q}$ becomes a second degree polynomial of $R^{*}$ with the maximum near the mid-point of the range of $R^{*}$, i.e., $\sqrt{3} / 4$. This result is very similar to the one obtained for dc PWM [15]. For the naturally-sampled dc PWM, the ripple magnitude is equal to $M \cdot \tau \cdot(1-\tau)$, and $M=(T / 2)\left(V_{\mathrm{dc}} / L_{a}\right)$. In the dc case, the normalized effective value of the PWM input voltage (which is the value of the PWM input voltage normalized by magnitude of the carrier sawtooth function) at the switching time $T k+\tau$ is equal to the duty ratio $\tau$. In dc/dc converters, the "sampling" instants $T k+\tau$ occur when the modulating input voltage equals the sawtooth carrier signal.

For the ac PWM case, the $\Delta i_{4,1}$ ripple magnitude is $M\left(V_{d}^{*}+j V_{q}^{*}\right) /\left(V_{\mathrm{dc}}\right) \tau_{z}$, where $\tau_{z}=1-\tau_{1}-\tau_{2}, M=$ $\left(T_{0} / 2\right)\left(V_{\mathrm{dc}} / L\right)$ and $V_{d}^{*}+j V_{q}^{*}$ is the effective value of the ac PWM reference voltage, which is the value of the PWM input voltage at the sampling instants $T_{0} k+t_{s}$. In contrast with the naturally-sampled dc PWM, the voltage space ac PWM is digitally implemented and the sampling instants $T_{0} k+t_{s}$ can be selected with $t_{s}$ as a design parameter. While there is a difference in implementation of these two PWM strategies, the magnitude of the current ripple obeys a similar relationship. 


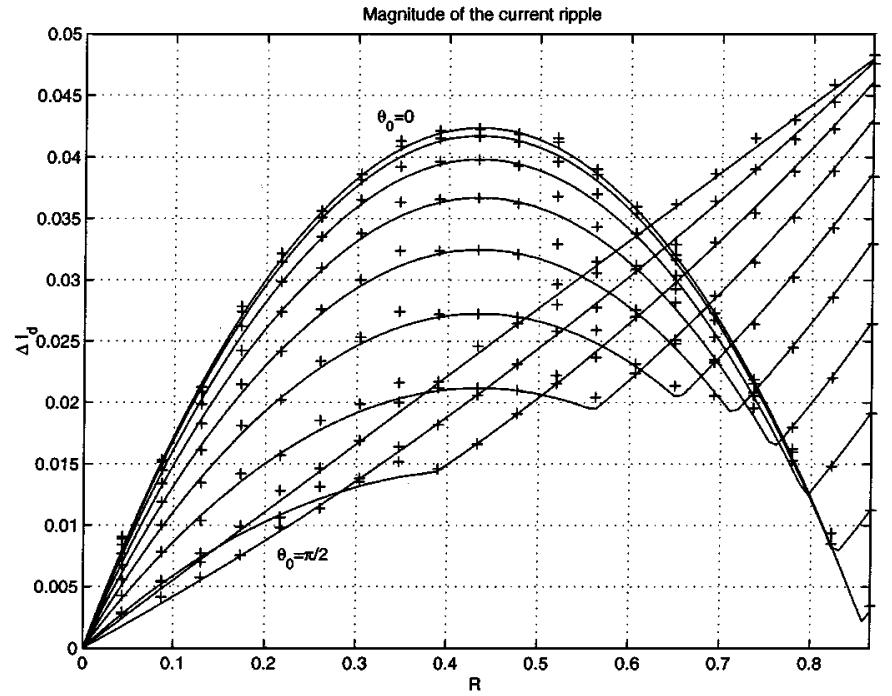

Fig. 6. Magnitude of $d$-current ripple normalized by $V_{\mathrm{cc}}$ as a function of $R^{*}$ and $\theta_{0}\left(N=24, n=1, M / V_{\mathrm{dc}}=0.195\right)$, theoretical (-) obtained by formula $\max \left[\left|\Delta i_{4,1}^{d}\right|,\left|\Delta i_{3,2}^{d}\right|\right]$ and from simulation of the full nonlinear closed-loop system $(+)$.

Next, we turn our attention to $\Delta i_{3,2}^{d}$ and $\Delta i_{3,2}^{q}$; for $\theta_{0}=0$ or $\theta_{0}=\pi$

$$
\begin{aligned}
\Delta i_{3,2}^{d}= & M \frac{2}{\sqrt{3}} R^{*} \sin \left(\frac{2 n+1}{N} \pi\right) \\
& \times\left(\sin \left(\frac{2 n+1}{N} \pi+\frac{\pi}{6}\right)-R^{*}\right) \\
\Delta i_{3,2}^{q}= & M \frac{2}{\sqrt{3}} R^{*} \sin \left(\frac{2 n+1}{N} \pi\right) \\
& \times \cos \left(\frac{2 n+1}{N} \pi+\frac{\pi}{6}\right) .
\end{aligned}
$$

The first equation is a second degree polynomial in $R^{*}$, with maximum

$$
\Delta i_{32, \max }^{d}=\frac{M}{2 \sqrt{3}} \sin \left(\frac{2 n+1}{N} \pi\right) \sin \left(\frac{2 n+1}{N} \pi+\frac{\pi}{6}\right)^{2} .
$$

The absolute maximum $\max _{n}\left[\Delta i_{32, \max }^{d}(n)\right]=M /(2 \sqrt{3})$ $\sin ((\pi / 3)-(\pi / N)) \cos (\pi / N)^{2}$ is reached at the last subinterval $n=N / 6-1$ and is less than $M / 4$. The second equation is a linear function of $R^{*}$, with the maximum at $R^{*}=\sqrt{3} / 2$ equal to

$$
\Delta i_{32, \max }^{q}=M \sin \left(\frac{2 n+1}{N} \pi\right) \cos \left(\frac{2 n+1}{N} \pi+\frac{\pi}{6}\right) .
$$

For different subintervals the absolute maximum $\max _{n}$ $\left[\Delta i_{32, \max }^{q}(n)\right]=M / 4$ is achieved in the middle subinterval $n=(N / 6-1) / 2$.

The same analysis can be performed for $\theta_{0}=$ $\pm(\pi / 2)$, when $\Delta i_{4,1}^{d}=0, \max _{n}\left[\Delta i_{41, \max }^{q}(n)\right]=$ $M / 4, \max _{n}\left[\Delta i_{32, \max }^{d}(n)\right]=M / 4$ and $\max _{n}\left[\Delta i_{32, \max }^{q}(n)\right]<$ $M / 4$. Finally, we conclude that current ripple magnitude is always less or equal to $M / 4$.

The results are summarized in Figs. 6-9. Figs. 6 and 7 show the magnitude of the current ripple for a specific subinterval, as

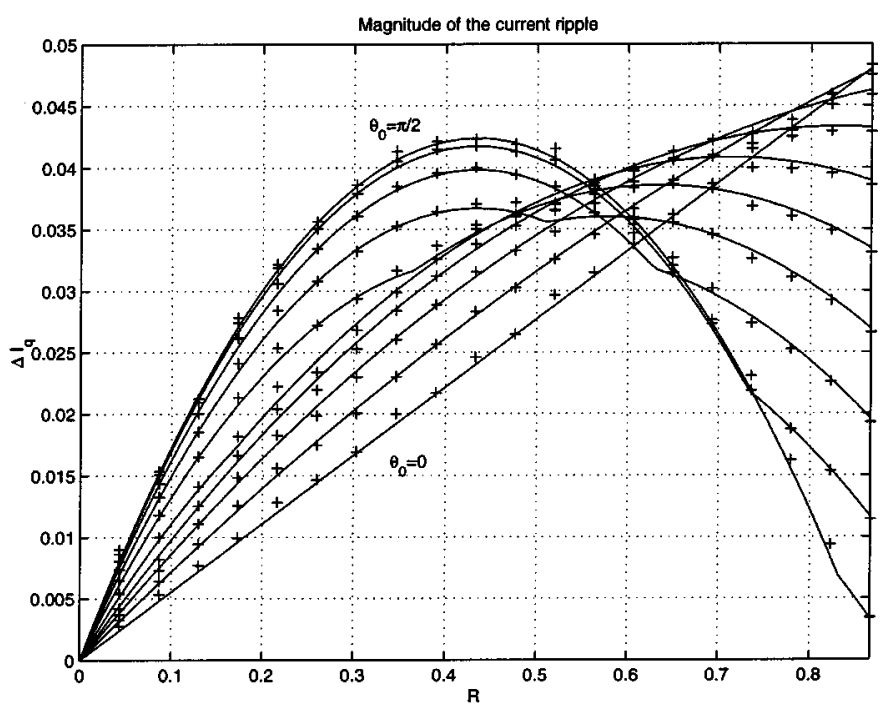

Fig. 7. Magnitude of $q$-current ripple normalized by $V_{\mathrm{dc}}$ as a function of $R^{*}$ and $\theta_{0}\left(N=24, n=1, M / V_{\mathrm{dc}}=0.195\right)$, theoretical $(-)$ obtained by formula $\max \left[\left|\Delta i_{4,1}^{q}\right|,\left|\Delta i_{3,2}^{q}\right|\right]$ and from simulation of the full nonlinear closed-loop system $(+)$.

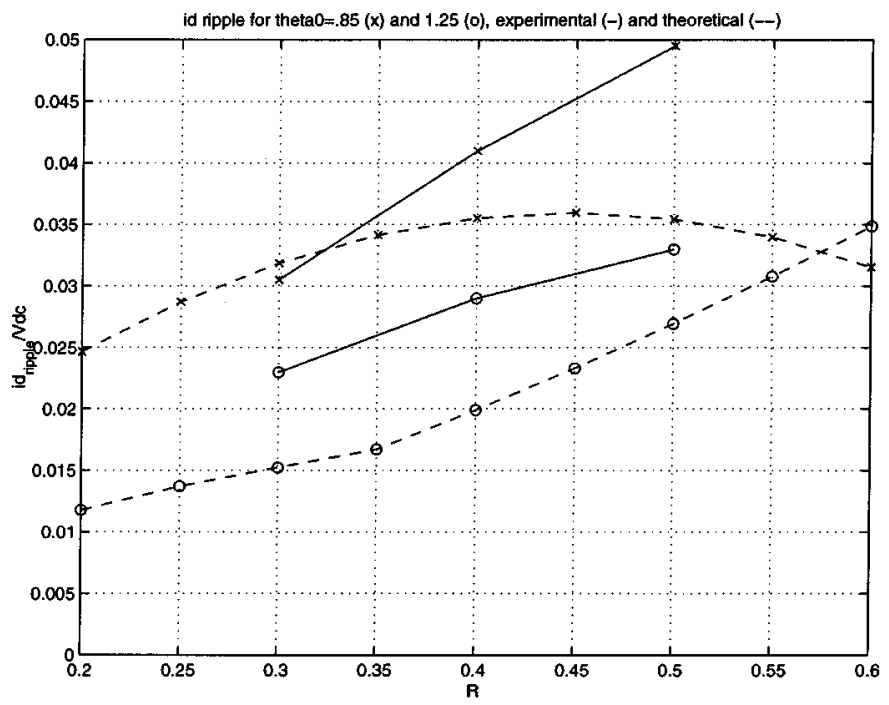

Fig. 8. Magnitude of $d$-current ripple normalized by $V_{\mathrm{cc}}$ as a function of $R^{*}$ and $\theta_{0}$, in the same conditions as Fig. 6, experimental (solid line) and theoretical (dashed line). The theoretical curves were adjusted by the variations of the inductances due to saturation.

a function of $R^{*}$ and $\theta_{0}$, computed from (26)-(29) and compared to results obtained by simulation of the full nonlinear closed-loop system. Fig. 8 shows experimental results, which are compared with the theoretical expressions mentioned above. In this case, due to saturation effects, the value of $M$ is not constant, and had to be adjusted in order to reflect the change in the inductances as the currents increase. Finally, in Fig. 9 we show the maximum of the $d$-current ripple over all subintervals $n=0, \ldots, N / 6-1$. Note the similarity with Fig. 6 ; the same holds for the $q$-current ripple.

\section{Closed-Loop PWM Gain}

If the PWM input (reference) voltage is constant, the closed-loop PWM gain is equivalent to the open-loop gain. 


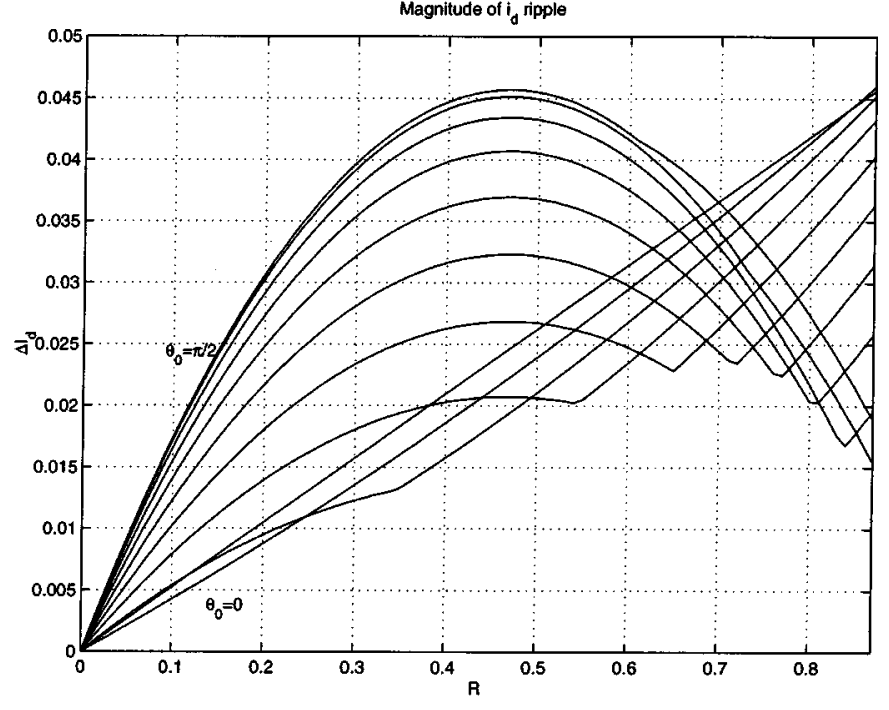

Fig. 9. Maximum magnitude of $d$-current ripple normalized by $V_{\mathrm{cc}}$ as a function of $R^{*}$ and $\theta_{0}$, over all subinterval $n=0, \ldots, N / 6-1$.

However, in the closed-loop system, current ripple may appear in the input of the PWM due to the proportional part of the controller. If in a digital implementation a $T_{0}$-periodic input of PWM $V_{d}^{*}$ (the analysis that follows applies equally to $q$ axis voltage and current) is sampled with period $T_{0}$, then this is equivalent to a constant PWM input reference voltage $V_{d}^{*}=V_{d}^{*}\left(k T_{0}+t_{s}\right)$, where $t_{s}$ is the sampling instance. The output $d$ voltage of the PWM, will be proportional to $V_{d}^{*}\left(k T_{0}+t_{s}\right)$, but not necessary to the average of the $V_{d}(t)^{*}$. If $t_{s}$ is chosen such that $V_{d}^{*}\left(k T_{0}+t_{s}\right)$ is equal to the averaged value of the $V_{d}(t)^{*}$, then the closed-loop PWM gain is again equal to the open-loop gain. To determine the closed-loop gain, $V_{d}^{*}\left(k T_{0}+t_{s}\right)$ and $V_{q}^{*}\left(k T_{0}+t_{s}\right)$ are required first. For a closed-loop system

$$
\begin{aligned}
& V_{d}^{*}(t)=-\omega L i_{q}^{*}-k_{p 1} i_{d}(t) \\
& V_{q}^{*}(t)=\omega \Phi+k_{p 2} i_{q}^{*}-k_{p 2} i_{q}(t) .
\end{aligned}
$$

Assuming that $\omega, i_{q}$ and $i_{q}^{*}$ vary slowly over a switching period

$$
\begin{aligned}
V_{d}^{*}(t) & =\bar{V}_{d}^{*}+\tilde{V}_{d}^{*}(t)=\left(-\omega L i_{q}^{*}-k_{p 1} \bar{i}_{d}\right)-k_{p 1} \tilde{i}_{d}(t) \\
V_{q}^{*}(t) & =\bar{V}_{q}^{*}+\tilde{V}_{q}^{*}(t)=\left(\omega \Phi+k_{p 2} i_{q}^{*}-k_{p 2} \bar{i}_{q}\right)-k_{p 2} \tilde{i}_{q}(t)
\end{aligned}
$$

where voltages and currents are represented as a sum of averaged value (denoted with a bar over the symbol) and ripple (denoted with a tilde over the symbol). The closed-loop gain then is

$$
\begin{aligned}
K_{\mathrm{PWM}, d}^{c . l .} & =\frac{\bar{V}_{d}}{\bar{V}_{d}^{*}}=\frac{V_{d}^{*}\left(k T_{0}+t_{s}\right)}{\bar{V}_{d}^{*}} \\
& =1+\frac{\tilde{V}_{d}^{*}\left(k T_{0}+t_{s}\right)}{\bar{V}_{d}^{*}}=1+G_{d} \frac{\tilde{i}_{d}\left(k T_{0}+t_{s}\right)}{\bar{V}_{d}^{*}}
\end{aligned}
$$

where $G_{d}=k_{p 1}$ (proportional gain of the current regulator, in terms of Fig. 1). The same equation applies to the $K_{\text {PWM, }}^{\mathrm{c} \text {.l. }}$, where $G_{q}=k_{p 2}$. In the above equation the open-loop gain is assumed to be equal to 1 , as it was shown previously that the error introduced by this approximation is very small. Now, our aim is to find $\tilde{i}\left(k T_{0}+t_{s}\right)$. It was shown previously that the shape of the current ripple is quite elaborate. Thus, only two practically relevant sampling strategies will be examined: 1) sampling at the midpoint of the PWM cycle $\left(t_{s} / T_{0}=-1 / 2\right)$, and 2) sampling at the beginning of the PWM cycle $\left(t_{s} / T_{0}=\right.$ 0 ). The expressions for $\tilde{i}\left(k T_{0}-T_{0} / 2\right)$ and $\tilde{i}\left(k T_{0}\right)$ are derived in Appendix B.

Integration of (13), (15) leads to

$$
\begin{aligned}
\frac{S_{d}^{1}-S_{d}^{3}}{2}= & V_{\mathrm{dc}} \sin \left(\frac{2 n+1}{N} \pi-\theta_{0}\right) \\
& \times\left(\cos \left(\tau_{1} \frac{\pi}{N}\right)-\cos \left(\tau_{2} \frac{\pi}{N}\right)\right) \\
\frac{S_{q}^{1}-S_{q}^{3}}{2}= & V_{\mathrm{dc}} \cos \left(\frac{2 n+1}{N} \pi-\theta_{0}\right) \\
& \times\left(\cos \left(\tau_{1} \frac{\pi}{N}\right)-\cos \left(\tau_{2} \frac{\pi}{N}\right)\right) .
\end{aligned}
$$

After substituting this into (62), (63) and taking the first two elements of Taylor series expansion, the following approximation is obtained

$$
\begin{aligned}
\tilde{i}\left(k T_{0}-T_{0} / 2\right) \approx & V_{\mathrm{dc}}\left(\tau_{2}^{2}-\tau_{1}^{2}\right)\left(\frac{\pi}{N}\right)^{2} \frac{T_{0}}{L} \\
& \times \sin \left(\frac{2 n+1}{N} \pi-\theta_{0}\right)\left(\tau_{1} / 2+\tau_{2}\right) \\
\tilde{i}\left(k T_{0}\right) \approx & V_{\mathrm{dc}}\left(\tau_{2}^{2}-\tau_{1}^{2}\right)\left(\frac{\pi}{N}\right)^{2} \frac{T_{0}}{L} \\
& \times \sin \left(\frac{2 n+1}{N} \pi-\theta_{0}\right)\left(\tau_{1} / 2+\tau_{z}\right)
\end{aligned}
$$

From these equations, it follows that the error associated with choice of the sampling instance $t_{s} / T_{0}=-1 / 2$ is larger then the error associated with choice of the sampling instance $t_{s} / T_{0}=0$ if $\tau_{2}>\tau_{z}$, or equivalently if

$$
R^{*}>\frac{\sqrt{3}}{2} \frac{1}{2 \sin \left(\frac{2 n+1}{N} \pi\right)+\sqrt{3} \cos \left(\frac{2 n+1}{N} \pi\right)} \approx \frac{\sqrt{3}}{4} .
$$

The emerging rule is: if $R^{*}<\sqrt{3} / 4$, then it is better to sample at the midpoint $k T_{0}-T_{0} / 2$; if $R^{*}>\sqrt{3} / 4$ then sampling at the beginning of the PWM cycle $k T_{0}$ provides smaller error. In either case, the error in current measurements is less then $V_{\mathrm{dc}}(\pi / N)^{2}\left(T_{0} / L\right)$. This error is inversely proportional to the basic PWM frequency $\omega_{0}=2 \pi / T_{0}$ and to the square of the number of PWM cycles per fundamental $N=\left(T / T_{0}\right)$. In other words, the error in current due to sampling at the middle $\left(k T_{0}\right)$ or beginning $\left(k T_{0}-T_{0} / 2\right)$ of the PWM basic cycle is proportional to the maximal ripple magnitude $V_{\mathrm{dc}}\left(T_{0} / 8 L\right)$ and to the voltage pulse asymmetry $S^{1}-S^{3}$ (which, in turn, is proportional to $\left.(\pi / N)^{2}\right)$.

In the worst-case sampling, when current $i(t)$ achieves its maximal or minimum at $k T_{0}+t_{s}$, the deviation of the PWM gain will be maximal. In this case the error in current due to sampling (difference between averaged current and sampled one) can equal $\Delta i_{32, \max }$ or $\Delta i_{41, \max }$, with the maximum equal to the current ripple magnitude $V_{\mathrm{dc}}\left(T_{0} / 8 L\right)$. 

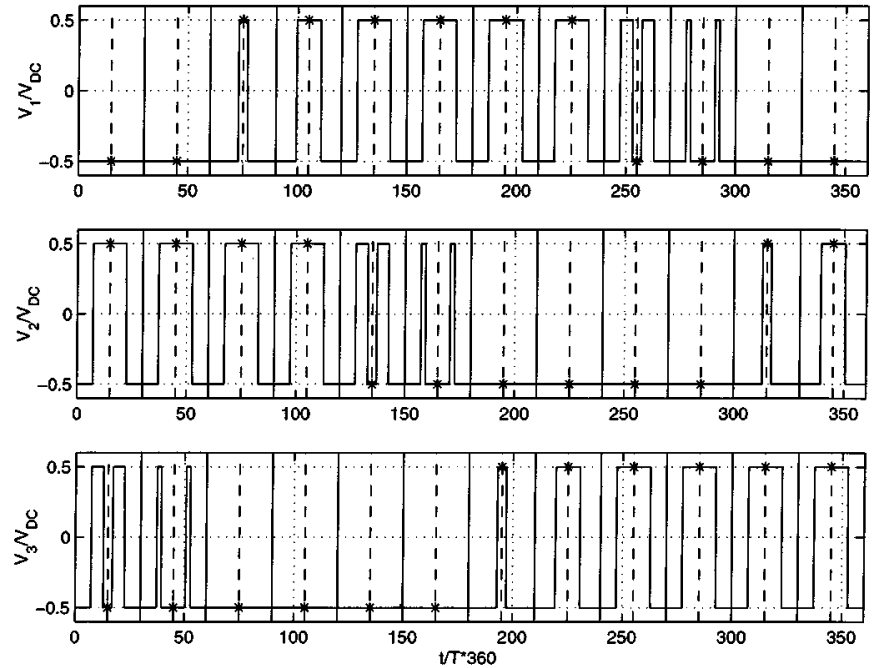

Fig. 10. PWM 3-phase line voltages.
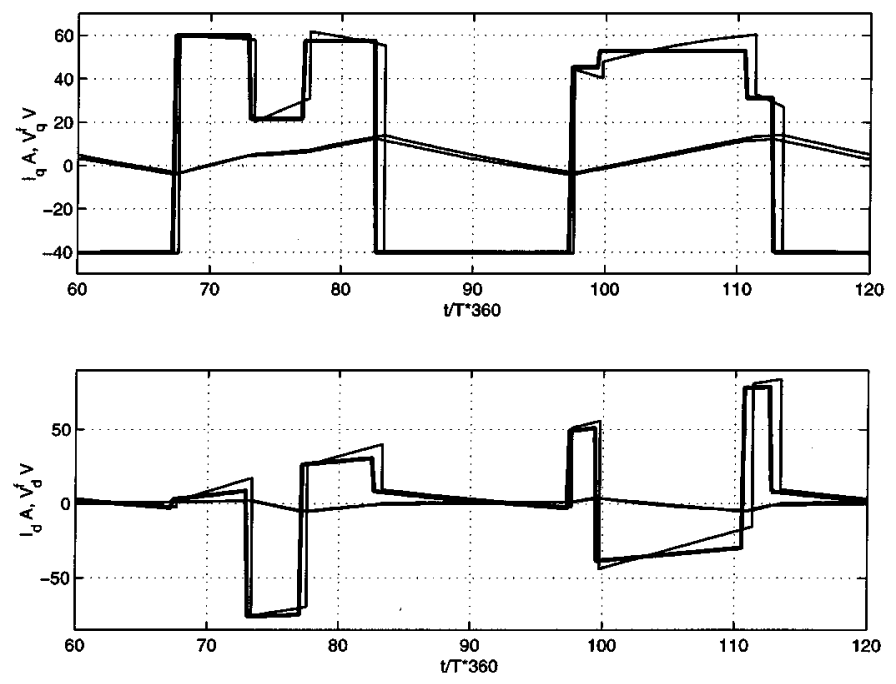

Fig. 11. Output of the PWM in $d-q$ coordinates, actual (thin lines) and approximated (bold lines).

\section{EXAMPLE}

The effectiveness of the approximations derived in this paper is illustrated on a fractional horse power PM synchronous motor. The parameters of the motor and the controller in simulations and experiments are: $R_{a}=1.25 \Omega ; L=6.7 \mathrm{mH} ; J=2.0 \cdot 10^{-2}$ $\mathrm{kg}-\mathrm{m}^{2} ; B=1.0 \cdot 10^{-3} \mathrm{Nms} ; T_{\text {load }}=0.8 \mathrm{Nm} ; \Phi=0.4 \mathrm{Nm} / \mathrm{A}$; $V_{\mathrm{dc}}=60 \mathrm{~V} ; k_{p 1}=1 ; k_{p 2}=1$. The system was simulated in Simulink using a full nonlinear model, i.e., without any simplifications. The switching frequency is such that there are 12 basic cycles within each period of fundamental frequency (i.e., $T=12 T_{0}$ or $N=12$ ). The normalized line voltages are shown in Fig. 10, while Fig. 11 shows voltages $V_{d}$ and $V_{q}$ and currents $i_{d}$ and $i_{q}$ both for complete model, and for approximate model derived in this paper. An experiment was performed under the same conditions, and the waveforms recorded, which are consistent with the simulation, are shown in Fig. 12.

The approximation error for currents (Fig. 11) is quite small, indicating that our technique can be used to estimate magnitude and ripple behavior. The currents are feed back to the controller,
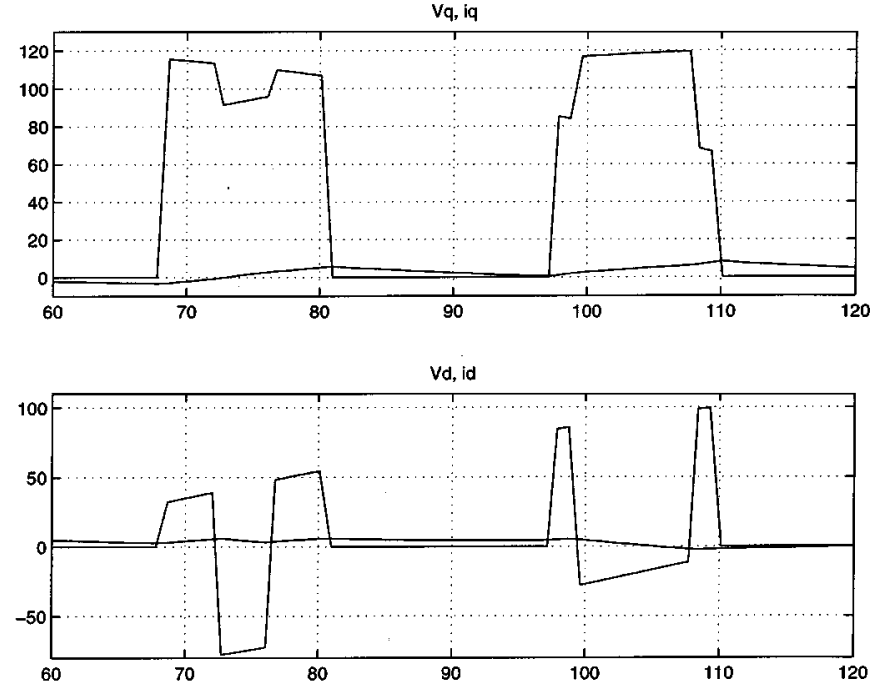

Fig. 12. Output of the PWM in $d-q$ coordinates, experimental results.

causing ripple in $V_{d}^{*}$ and $V_{q}^{*}$. Because of this effect, the average value of the PWM input voltages $\bar{V}_{d}^{*}, \bar{V}_{q}^{*}$ and actual measured voltages $V_{d}^{*}\left(k T_{0}+t_{s}\right)$ and $V_{q}^{*}\left(k T_{0}+t_{s}\right)$ can differ, and consequently the average PWM gain can deviate from ideal. In our example, the average $\bar{V}_{q}^{*} \approx 42.4 \mathrm{~V}$. The maximum possible magnitude of the ripple $\Delta V_{q, \max }=k_{p 2} \Delta i_{\max }^{q}$ is $5.86 \mathrm{~V}$, and the gain of PWM along $q$ axis $K_{\mathrm{PWM}, q}^{\mathrm{C} . \mathrm{l}}=1+k_{p 2} \Delta i_{\max }^{q} / \bar{V}_{q}^{*}=1.14$ which corresponds approximately to $14 \%$ deviation from nominal. For the $d$ axis, $\bar{V}_{d}^{*} \approx-2.4 \mathrm{~V}$, and the maximum possible magnitude of the ripple $\Delta V_{d, \max }=k_{p 1} \Delta i_{\max }^{q}$ has the same value of $5.86 \mathrm{~V}$. Thus the gain of PWM along $d$ axis may achieve $K_{\mathrm{PWM}, d}^{\text {c.l. }}=1+k_{p 1} \Delta i_{\max }^{d} / \bar{V}_{d}^{*}=3.44$, which corresponds approximately to $244 \%$ deviation from nominal. To interpret this last result, note that if the input voltage is aligned with the $q$ axis, then $V_{d}^{*}$ is equal to zero, and any $\tilde{V}_{d}^{*}\left(k T_{0}+t_{s}\right) \neq 0$ will produce infinite error in the PWM gain along the $d$ axis. The example illustrates the fact that the relative deviation of the PWM gain may become large when the input voltage $V^{*}$ is close to one of the axes (which is the case in our example when $\theta^{*}=93.2^{\circ}$ ).

\section{CONCLUSION}

This paper presents analytical results, numerical simulations, and experiments that quantify effects of low switching frequency on ac drives. In particular, analysis of the current ripple pattern is provided, and open- and closed-loop gains of the PWM block are evaluated. These analytical expressions quantify the deviations from the idealized models used in control studies, and provide design guidelines in terms of system quantities, such as the relative and absolute switching frequency and converter and machine parameters. Presented formulas are verified by both simulations and laboratory experiments. While the PM synchronous motor is used as an example throughout, the methodology is applicable to design and control of other types of ac drives and active power filters.

\section{APPENDIX A}

To find the piecewise-constant approximation of the voltage $V$, each subinterval length of $T / N$ is divided into five smaller 
intervals within which the voltage will be approximated by constants $V^{\ell}(n), \ell=0, \ldots, 4$, each equal to the averaged voltage within the corresponding interval. From piecewise-constant approximation (25) the peak current values are

$$
\begin{aligned}
& i_{1}=\frac{V^{0}-R_{a} \bar{i}}{L} \frac{\tau_{z} T_{0}}{2}+i_{0} \\
& i_{2}=\frac{V^{1}-R_{a} \bar{i}}{L} \frac{\tau_{1} T_{0}}{2}+i_{1} \\
& i_{3}=\frac{V^{2}-R_{a} \bar{i}}{L} \tau_{2} T_{0}+i_{2} \\
& i_{4}=\frac{V^{3}-R_{a} \bar{i}}{L} \frac{\tau_{1} T_{0}}{2}+i_{3} \\
& i_{5}=\frac{V^{0}-R_{a} \bar{i}}{L} \frac{\tau_{z} T_{0}}{2}+i_{4} .
\end{aligned}
$$

For the considered switching pattern $V^{0}=0, V^{1}=$ $2 S^{1} / \tau_{1}, V^{2}=S^{2} / \tau_{2}, V^{3}=2 S^{3} / \tau_{1}, V^{4}=0$ and $\bar{i}=\left(S^{1}+S^{2}+S^{3}\right) / R_{a}$, where $S^{\ell}$ are given by (13)-(15). Then

$$
\begin{aligned}
& \Delta i_{1,0}=-\frac{1}{2} \frac{S(n)}{L} \frac{\tau_{z} T_{0}}{2} \\
& \Delta i_{2,1}=-\frac{1}{2}\left[\frac{S(n)}{L} \frac{\tau_{1} T_{0}}{2}-\frac{S^{1}(n)}{L} T_{0}\right] \\
& \Delta i_{3,2}=-\frac{1}{2}\left[\frac{S(n)}{L} \tau_{2} T_{0}-\frac{S^{2}(n)}{L} T_{0}\right] \\
& \Delta i_{4,3}=-\frac{1}{2}\left[\frac{S(n)}{L} \frac{\tau_{1} T_{0}}{2}-\frac{S^{3}(n)}{L} T_{0}\right] \\
& \Delta i_{0,4}=-\frac{1}{2} \frac{S(n)}{L} \frac{\tau_{z} T_{0}}{2}
\end{aligned}
$$

where $\Delta i_{\ell, k}=\left(i_{\ell}-i_{k}\right) / 2$. The ripple magnitude is the maximum of $\left|\Delta i_{4,1}\right|$ and $\left|\Delta i_{3,2}\right|$

$$
\begin{aligned}
\Delta i_{4,1} & =-\frac{1}{2} \frac{S(n)}{L} \tau_{z} T_{0} \\
\Delta i_{3,2} & =-\frac{1}{2}\left[\frac{S(n)}{L} \tau_{2} T_{0}-\frac{S^{2}(n)}{L} T_{0}\right] .
\end{aligned}
$$

\section{APPENDIX B}

First, an explicit equation for $i_{\ell}$ is needed; since (46)-(50) are not linearly independent, one more equation should be added to solve for $i_{\ell}: \bar{i}=\int_{k T_{0}}^{k T_{0}+T_{0}} i(t) d t$. After integrating, we obtain

$$
\begin{aligned}
\bar{i}=\frac{i_{1}+i_{0}}{4} \tau_{z}+\frac{i_{2}+i_{1}}{4} \tau_{1}+ & \frac{i_{3}+i_{2}}{2} \tau_{2} \\
& +\frac{i_{3}+i_{4}}{4} \tau_{1}+\frac{i_{4}+i_{0}}{4} \tau_{z} .
\end{aligned}
$$

Then, $i_{\ell}$ can be found from the following equation:

$$
\left[\begin{array}{c}
\Delta i_{1,0} \\
\Delta i_{2,1} \\
\Delta i_{3,2} \\
\Delta i_{4,3} \\
\Delta i_{0,4} \\
\bar{i}
\end{array}\right]=\left[\begin{array}{ccccc}
-1 / 2 & 1 / 2 & 0 & 0 & 0 \\
0 & -1 / 2 & 1 / 2 & 0 & 0 \\
0 & 0 & -1 / 2 & 1 / 2 & 0 \\
0 & 0 & 0 & -1 / 2 & 1 / 2 \\
1 / 2 & 0 & 0 & 0 & -1 / 2 \\
a_{0} & a_{1} & a_{2} & a_{3} & a_{4}
\end{array}\right]\left[\begin{array}{c}
i_{0} \\
i_{1} \\
i_{2} \\
i_{3} \\
i_{4}
\end{array}\right]
$$

where

$$
\begin{aligned}
& a_{0}=\frac{\tau_{z}}{2} \quad a_{1}=\frac{\tau_{z}+\tau_{1}}{4} \quad a_{2}=\frac{\tau_{1} / 2+\tau_{2}}{2} \\
& a_{3}=a_{2} \quad a_{4}=a_{1} \text {. }
\end{aligned}
$$

1) If current is sampled at $k T_{0}-T_{0} / 2$, then $i\left(k T_{0}-T_{0} / 2\right)=i_{0}$. Solving for $i_{0}(61)$ shown at the bottom of the page. Note that $2 \Delta i_{1,0}+\Delta i_{2,1}+\Delta i_{3,2}+\Delta i_{4,3}=0, a_{0}+2 a_{1}+2 a_{2}=1$, and $\tilde{i}\left(k T_{0}-T_{0} / 2\right)=i_{0}-\bar{i}$, then

$$
\tilde{i}\left(k T_{0}-T_{0} / 2\right)=-\left(\tau_{1} / 2+\tau_{2}\right) \frac{S^{1}-S^{3}}{2} \frac{T_{0}}{L} .
$$

2) If current is sampled at $k T_{0}$, then $i\left(k T_{0}\right)=\left(i_{2}+i_{3}\right) / 2$

$$
i\left(k T_{0}\right)=\Delta i_{1,0}+\Delta i_{2,1}+\Delta i_{3,2} / 2+i_{0}
$$

and $\tilde{i}\left(k T_{0}-T_{0} / 2\right)=\left(i_{2}+i_{3}\right) / 2-\bar{i}$

$$
\tilde{i}\left(k T_{0}\right)=\left(1-\tau_{1} / 2-\tau_{2}\right) \frac{S^{1}-S^{3}}{2} \frac{T_{0}}{L} .
$$

\section{REFERENCES}

[1] J. Holtz and A. Khambadkone, "Low switching frequency and high dynamic pulsewidth modulation based on field-orientation for high-power inverter drive," IEEE Trans. Power Electron., vol. 7, pp. 627-632, Oct. 1992.

[2] J. Holtz and E. Bube, "Field oriented asynchronous pulse-width modulation for high performance ac machine drives operating at low switching frequency," Proc. IEEE Industry Appl. Soc. Annu. Meeting, pp. 412-417, 1988.

[3] N. Mohan, T. M. Undeland, and W. P. Robbins, Power Electronics, 2nd ed. New York: Wiley, 1995.

[4] J. Holtz, "Pulsewidth modulation-A survey," IEEE Trans. Power Electron., vol. 39, pp. 410-420, Oct. 1992.

[5] J. Holtz, W. Lotzkat, and A. M. Khambadkone, "On continuous control of PWM inverters in the overmodulation range including the six-step mode," IEEE Trans. Power Electron., vol. 8, pp. 546-553, Oct. 1993.

[6] J. Holtz and B. Beyer, "Optimal pulsewidth modulation for ac servos and low-cost industrial drives," IEEE Trans. Ind. Applicat., vol. 30, pp. 1039-1047, July 1994.

[7] I. Boldea, Electric Machine Dynamics. New York: MacMillan, 1986.

[8] A. M. Trzynadlowski and S. Legowski, "Minimum-loss vector PWM strategy for three-phase inverters," IEEE Trans. Power Electron., vol. 9, pp. 26-34, Jan. 1994.

[9] P. Lammert, J. Holtz, and W. Lotzkat, "High-speed drive system with ultrasonic MOSFET PWM inverter and single-chip microprocessor control,” Proc. IEEE Industry Appl. Soc. Annu. Meeting, pp. 12-17, 1986.

[10] H. C. Skudelny, H. W. Van der Broeck, and G. Stanke, "Analysis and realization of a pulse width modulator based on voltage space vectors," Proc. IEEE Industry Appl. Soc. Annu. Meeting, pp. 244-251, 1986.

[11] P. Vas, Electrical Machines and Drives. A Space-Vector Theory Approach. New York: Oxford Univ. Press, 1992.

$$
i_{0}=\frac{\bar{i}-a_{1}\left(2 \Delta i_{1,0}+\Delta i_{2,1}+\Delta i_{3,2}+\Delta i_{4,3}\right)-a_{2}\left(2 \Delta i_{1,0}+2 \Delta i_{2,1}+\Delta i_{3,2}\right)}{a_{0}+2 a_{1}+2 a_{2}} .
$$


[12] J. Holtz, "Pulse width modulation for electrical power converters," in Power Electronics and Variable Frequency Drives, B. K. Base, Ed. New York: IEEE Press, 1997.

[13] D. W. Novotny and T. A. Lipo, Vector Control and Dynamics of AC Drives. New York: Oxford Univ. Press, 1996.

[14] W. Leonhard, Control of Electric Drives. New York: Springer-Verlag, 1985.

[15] T. A. Sakharuk, B. Lehman, A. M. Stanković, and G. Tadmor, "Effects of finite switching frequency and delay on PWM controlled systems," IEEE Trans. Circuits Syst., vol. 47, pp. 555-567, Apr. 2000.

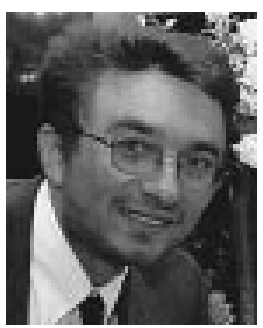

Timoor A. Sakharuk was born in Leningrad, Russia in 1965. He received the Diploma in electrical engineering from Leningrad Institute of Fine Mechanics and Optics, Leningrad, Russia, the Diploma in mathematics from Leningrad State University, Leningrad, Russia, and the Ph.D. degree in electrical engineering from Northeastern University, Boston, MA, in 1988, 1992, and 1999, respectively.

From 1998 to 2000, Dr. Sakharuk was working as an Engineer at the R\&D Carrier Systems Group of the 3Com Corporation, Boston, MA. At present, he is a senior DSP Engineer at the Network Communications Group of Intel Corporation, Boston, MA. His current research interests are in signal processing and communication.

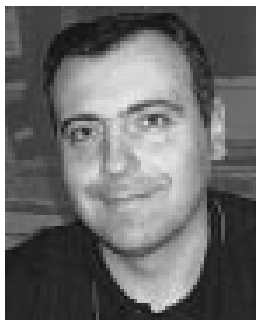

Aleksandar M. Stankovic obtained the Dipl.Ing. degree and the M.S. degree, both from the University of Belgrade, Yugoslavia in 1982, and 1986, respectively, and the Ph.D. degree from Massachusetts Institute of Technology, Cambridge, MA, in 1993, all in electrical engineering.

He has been with the Department of Electrical and Computer Engineering at Northeastern University, Boston, MA, since 1993, presently as an Associate Professor. His research interests are in modeling, analysis, estimation and control of energy processing systems.

In 2000/01 Dr. Stankovic served as chair of the IEEE Circuits and Systems Society Technical Committee on Power Systems and Power Electronic Circuits. He presently serves as an Associate Editor for the IEEE TRANSACTIONS on Control System Technology and for IEEE TRANSACtions ON POWER SYSTEMS.

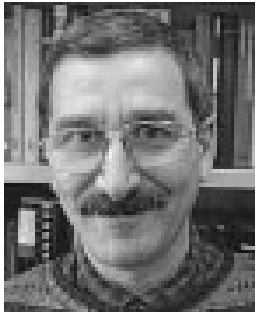

Gilead Tadmor (M'87-SM'92) received the B.Sc. degree from Tel Aviv University, Tel Aviv, Israel, in 1977, and the M.Sc. and Ph.D. degrees, from the Weizmann Institute of Science, Israel, in 1979, and 1984, respectively, all in mathematics (systems and control).

$\mathrm{He}$ joined the Department of Electrical and Computer Engineering at Northeastern University, Boston, MA, in 1989, where he is currently an Associate Professor. His recent active interests include nonlinear control, with applications in power electronics, power systems, electric motor drives, mechanical systems and fluid flow control. Dr. Tadmor currently serves as an Associate Editor with the Control Systems Society Conference Editorial Board.

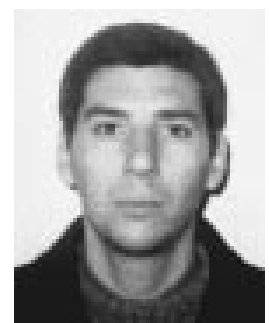

Gabriel Eirea received the electrical engineering degree and the M.Sc. degree in electrical engineering, from the Universidad de la Republica, Monterides, Uruguay, and Northeastern University, Boston, MA, in 1997, and 2001, respectively. He is currently working toward the Ph.D. degree at the University of California, Berkeley. His research interests include systems theory, embedded control, and applications involving hybrid dynamical systems. 